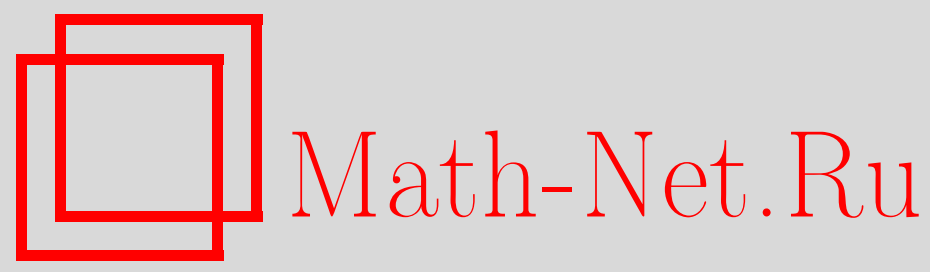

О. В. Лычагина, Нормальные формы пуассоновых структур, Матем. заметки, 1997, том 61, выпуск 2, 220-235

DOI: https://doi.org/10.4213/mzm1495

Использование Общероссийского математического портала Math-Net.Ru подразумевает, что вы прочитали и согласны с пользовательским соглашением http://www. mathnet.ru/rus/agreement

Параметры загрузки:

IP: 3.85 .183 .62

26 апреля 2023 г., 16:35:28

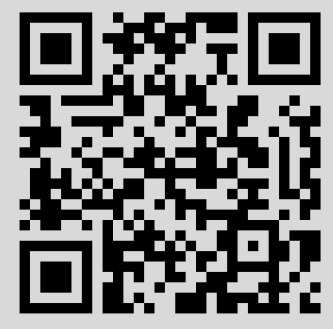




\title{
НОРМАЛЬНЫЕ ФОРМЫ ПУАССОНОВЫХ СТРУКТУР
}

\author{
О.В. Лычагина
}

\begin{abstract}
В работе найдены формальные нормальные формы пуассоновых структур. Как следствие получены препятствия к формальной линеаризации пуассоновых структур, что дает обобщение теоремы Вейнстейна о линеаризуемости. В работе также введены и исследованы алгебры Ли-Склянина, отвечающие пуассоновым структурам с тривиальной линеаризацией.

Библиография: 8 названий.
\end{abstract}

Введение. В этой работе мы рассматриваем приложение пуассоновых когомологий к задаче формальной классификации вырожденных пуассоновых структур. Выбор таких структур основан на “теореме о расщеплении” А. Вейнстейна [1], которая сводит локальное изучение пуассоновых структур к изучению структур, обращающихся в нуль в фиксированной точке.

Мы показываем, что препятствия к линеаризации такой пуассоновой структуры лежат в группе вторых когомологий алгебры Ли, определяемой линейной частью этой пуассоновой структуры.

В случае, когда когомологии тривиальны (например, алгебра Ли является полупростой или редуктивной с одномерным центром) соответствующая пуассонова структура формально эквивалентна линейной.

Если алгебра Ли коммутативна, то следующий по порядку малости член пуассоновой структуры определяет на касательном пространстве структуру некоторой алгебры, которую мы назьваем алгеброй Ли-Склянина. Препятствия для эквивалентности данной пуассоновой структуры квадратичной лежат в группе когомологий этой алгебры.

Для нахождения нормальных форм вырожденных пуассоновых структур мы используем метод спектральных последовательностей, предложенньй в [1] для нахождения нормальных форм особенностей функций и в [2] для нахождения нормальных форм алгебр Ли векторных полей.

Мы рассматриваем комплекс для нахождения пуассоновых когомологий и в терминах спектральной последовательности, ассоциированной с некоторой фильтрацией этого комплекса, приводим нормальные формы вырожденных пуассоновьх структур.

\section{§1. Скобка Схоутена на алгебре поливекторных полей}

Пусть $M=M^{n}$ - гладкое $n$-мерное многообразие, $\mathscr{D}=\mathscr{D}(M)$ - модуль векторных полей на $M$. Обозначим через

$$
\mathscr{D}_{j}=\Lambda^{j}(\mathscr{D})=\underbrace{\mathscr{D} \wedge \cdots \wedge \mathscr{D}}_{j \text { раз }}
$$


модуль $j$-векторньг полей на $M, \mathscr{D}_{0}=A=C^{\infty}(M)$, и через $i_{f}$ - внутреннее дифференцирование на дифференциал $d f$ гладкой функции $f \in A$.

Пусть $\left[\mathscr{D}_{i}, \mathscr{D}_{j}\right] \subset \mathscr{D}_{i+j-1}-$ скобка Схоутена на алгебре поливекторных полей. Напомним ее основные свойства:

1) $\|[f, X]=\llbracket[X, f]=i_{f} X, f \in A, X \in \mathscr{D}_{i}$;

2) $i_{f} \llbracket X, Y \rrbracket=\| i_{f} X, Y \rrbracket+(-1)^{i} \llbracket\left[X, i_{f} Y \rrbracket\right.$, где $X \in \mathscr{D}_{i}, Y \in \mathscr{D}_{j}$;

3) $\left.\| X, Y]=(-1)^{i j} \| Y, X\right], X \in \mathscr{D}_{i}, Y \in \mathscr{D}_{j}$;

4) $\left\|[X, Y \wedge Z]=(-1)^{k}\right\|[X, Y] \wedge Z+(-1)^{i j} Y \wedge \|[X, Z], X \in \mathscr{D}_{i}, Y \in \mathscr{D}_{j}, Z \in \mathscr{D}_{k}$;

5) $[X, \| Y, Z] \mid]=(-1)^{k+1}\left[[[X, Y], Z]\left|+(-1)^{i j+1}\right|[Y, \|[X, Z]] \mid, X \in \mathscr{D}_{i}, Y \in \mathscr{D}_{j}\right.$, $Z \in \mathscr{D}_{k}$

6) любой диффеоморфизм $\varphi: M \rightarrow M$ индуцирует отображения

$$
\varphi_{*}^{(i)}: \mathscr{D}_{i} \rightarrow \mathscr{D}_{i}
$$

где отображение $\varphi_{*}^{(0)}=\varphi_{*}: \mathscr{D}_{0} \rightarrow \mathscr{D}_{0}$ задается формулой

$$
\varphi_{*}(f)=f \circ \varphi^{-1}, \quad f \in A \text {. }
$$

Отображение $\varphi_{*}^{(1)}: \mathscr{D}_{1} \rightarrow \mathscr{D}_{1}$ таково, что

$$
\varphi_{*}^{(1)}(X)=\left(\varphi^{*}\right)^{-1} \circ X \circ \varphi^{*},
$$

где $X \in \mathscr{D}_{1}$, а $\varphi_{*}^{(i)}=\Lambda^{i}\left(\varphi_{*}^{(1)}\right), i \geqslant 1,-$ внешние степени $\varphi_{*}^{(1)}$.

В этом случае $\varphi_{*}^{(i)}(f X)=\varphi_{*}(f) \varphi_{*}^{(i)}(X), f \in \mathscr{D}, X \in \mathscr{D}_{i}$, и отображения $\varphi_{*}^{(i)}, i \geqslant 1$, согласованы со скобкой Схоутена:

$$
\left.\left.\llbracket \varphi_{*}^{(i)}(X), \varphi_{*}^{(j)}(Y)\right]=\varphi_{*}^{(i+j-1)}(\llbracket X, Y]\right), \quad X \in \mathscr{D}_{i}, \quad Y \in \mathscr{D}_{j}
$$

\section{$\S 2$. Пуассонова структура на многообразии}

2.1. Каждое бивекторное поле $\nabla \in \mathscr{D}_{2}$ определяет скобку на алгебре функций:

$$
[f, g]_{\nabla}:=\langle\nabla, d f \wedge d g\rangle=i_{g} i_{f}(\nabla) .
$$

Теорема. Алгебра $C^{\infty}(M)$ является алгеброй Ли относительно $[\cdot, \cdot]_{\nabla}$ в том и только том случае, когда выполнено следующее условие:

$$
\| \nabla, \nabla]=0 .
$$

ОПРЕДЕЛЕНИЕ. Скажем, что на многообразии $M$ определена пуассонова cmpyкту$p a$, если задано такое бивекторное поле $\nabla \in \mathscr{D}_{2}$, что $\llbracket \nabla, \nabla \rrbracket=0$. Гладкое многообразие с заданной пуассоновой структурой назьвается пуассоновым.

Укажем явный вид скобки Схоутена $[[\nabla, \nabla]$ в локальных координатах.

Если $x_{1}, \ldots, x_{n}-$ система локальных координат на $M$ и

$$
\nabla=\sum_{i, j} a_{i j}(x) \frac{\partial}{\partial x_{i}} \wedge \frac{\partial}{\partial x_{j}}
$$

где $a_{i j}(x)$ - гладкие функции на рассматриваемой координатной окрестности, то $\llbracket \nabla, \nabla \rrbracket=\sum_{s<t<r} \sum_{k}\left(a_{s k}(x) \frac{\partial a_{t r}(x)}{\partial x_{k}}-a_{k r}(x) \frac{\partial a_{s t}(x)}{\partial x_{k}}-a_{k t}(x) \frac{\partial a_{s r}(x)}{\partial x_{k}}\right) \frac{\partial}{\partial x_{s}} \wedge \frac{\partial}{\partial x_{t}} \wedge \frac{\partial}{\partial x_{r}}$. 
2.2. Каждое бивекторное поле $\nabla \in \mathscr{D}_{2}$ определяет последовательность

$$
0 \longrightarrow \mathscr{D}_{0}=A \stackrel{\partial_{\nabla}}{\longrightarrow} \mathscr{D}_{1} \stackrel{\partial_{\nabla}}{\longrightarrow} \ldots \stackrel{\partial_{\nabla}}{\longrightarrow} \mathscr{D}_{i} \stackrel{\partial_{\nabla}}{\longrightarrow} \mathscr{D}_{i+1} \stackrel{\partial_{\nabla}}{\longrightarrow} \ldots \stackrel{\partial_{\nabla}}{\longrightarrow} \mathscr{D}_{n} \rightarrow 0
$$

где $\partial_{\nabla}(X):=(-1)^{i} \llbracket[X, \nabla]$ для $X \in \mathscr{D}_{i}$.

Из свойства 4) следует, что оператор $\partial_{\nabla}$ является дифференцированием степени 1 алгебры $\mathscr{D}_{*}=\sum_{i \geqslant 0} \mathscr{D}_{i}$, т.е.

$$
\partial_{\nabla}(X \wedge Y)=\partial_{\nabla}(X) \wedge Y+(-1)^{i} X \wedge \partial_{\nabla}(Y)
$$

для $X \in \mathscr{D}_{i}, Y \in \mathscr{D}_{j}$.

Кроме того, оператор $\partial_{\nabla}$ является также дифференцированием относительно скобки Схоутена, т.е.

$$
\left.\left.\partial_{\nabla}(\llbracket X, Y]\right)=\llbracket \partial_{\nabla}(X), Y\right] \mid+(-1)^{i} \llbracket\left[X, \partial_{\nabla}(Y)\right]
$$

для $X \in \mathscr{D}_{i}, Y \in \mathscr{D}_{j}$.

Последовательность (1) является комплексом в том и только том случае, когда $\nabla-$ пуассонова структура.

Когомологии комплекса (1) в члене $\mathscr{D}_{i}$ назьваются пуассоновыми когомологиями многообразия $M[3]$ и обозначаются

$$
H_{\nabla}^{i}(M)=\frac{\operatorname{Ker}\left(\partial_{\nabla}: \mathscr{D}_{i} \rightarrow \mathscr{D}_{i+1}\right)}{\operatorname{Im}\left(\partial_{\nabla}: \mathscr{D}_{i-1} \rightarrow \mathscr{D}_{i}\right)}
$$

2.3. Пусть $\nabla \in \mathscr{D}_{2}$ определяет пуассонову структуру на $M$. Рассмотрим оператор $\Gamma_{1}: \Omega_{1}(M) \rightarrow \mathscr{D}_{1}(M)$, действующий следующим образом: $\Gamma_{1}(\omega)=i_{\omega}(\nabla)$, где $\omega \in \Omega_{1}(M)$.

Положим $\Gamma_{i}=\Lambda^{i}\left(\Gamma_{1}\right), i \geqslant 1$. Тогда диаграмма

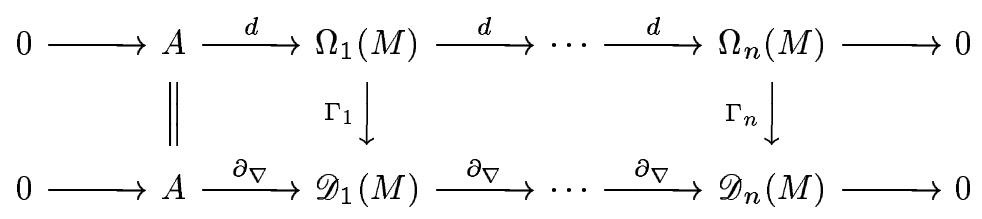

коммутативна (cp. [4], [5]).

2.4. Пуассонова структура $\nabla \in \mathscr{D}_{2}$ называется симплектической, если отображение $\Gamma_{1}: \Omega_{1}(M) \rightarrow \mathscr{D}_{1}(M)$ является изоморфизмом.

В этом случае задание бивекторного поля $\nabla$ эквивалентно выбору 2-формы $\omega_{\nabla} \in$ $\Omega_{2}(M)$, где $\omega_{\nabla}=\Gamma_{2}^{-1}(\nabla)$.

Из приведенной коммутативной диаграммы следует, что невырожденное бивекторное поле $\nabla$ определяет симплектическую структуру в том и только том случае, когда $d \omega_{\nabla}=0$.

Для симплектических многообразий пуассоновы когомологии совпадают с когомологиями де Рама [5]. 
2.5. Опишем пуассоновы когомологии в малых размерностях (cp. [4]). Для 0-мерных когомологий имеем

$$
H_{\nabla}^{0}(M)=\left\{f \in A \mid \partial_{\nabla}(f)=\llbracket f, \nabla \rrbracket=0\right\}
$$

или

$$
H_{\nabla}^{0}(M)=\left\{f \in A \mid[f, g]_{\nabla}=0 \forall g \in A\right\} .
$$

Таким образом, $H_{\nabla}^{0}$ совпадает с центром алгебры Ли $C^{\infty}(M)$, т.е. является подалгеброй функций Казимира.

Для описания 1-мерных пуассоновых когомологий заметим, что ядро

$$
\operatorname{Ker}\left(\partial_{\nabla}: \mathscr{D}_{1} \rightarrow \mathscr{D}_{2}\right)=\left\{X \in \mathscr{D}_{1} \mid L_{X}(\nabla)=0\right\}
$$

совпадает с алгеброй Ли всех пуассоновых векторных полей на $M$ (т.е. с алгеброй Ли инфинитезимальных симметрий пуассоновой структуры), а образ $\operatorname{Im}\left(\partial_{\nabla}: \mathscr{D}_{0} \rightarrow \mathscr{D}_{1}\right)$ совпадает с алгеброй Ли гамильтоновьх полей $\left\{X_{f}=i_{f}(\nabla) \mid f \in A\right\}$. Отметим, что множество гамильтоновых векторных полей - идеал в алгебре Ли всех пуассоновых полей, следовательно, $H_{\nabla}^{1}$ является фактор-алгеброй Ли всех пуассоновьх векторных полей по идеалу гамильтоновых векторных полей.

Для интерпретации $H_{\nabla}^{2}$ рассмотрим два вида путей на множестве всех пуассоновых структур:

- пути общего вида: $\nabla_{t} \in \mathscr{D}_{2}, t \in \mathbb{R}$, такие, что $\left[\nabla_{t}, \nabla_{t} \|=0, \nabla_{0}=\nabla\right.$;

- тривиальные пути, т.е. пути вида $\nabla_{t}=\left(\varphi_{t}^{(2)}\right)_{*}(\nabla)$, где $\varphi_{t}-$ некоторый путь в групше диффеоморфизмов $M$, и $\varphi_{0}=\mathrm{id}$.

Опишем теперь два вида инфинитезимальных деформаций, отвечающих выбранным пуассоновым путям.

Рассматривая касательные к указанным путям, введем следующие инфинитезимальные деформации:

- общие: $\nabla_{t}=\nabla_{0}+t \nabla^{\prime}+o(t)$;

- тривиальные: $\nabla_{t}=\nabla_{0}+t L_{Y}\left(\nabla_{0}\right)+o(t)$ для некоторого векторного поля $Y \in \mathscr{D}_{1}$.

Заметим, что условие пуассоновости $\nabla_{t}$ для общей деформации, рассматриваемоепо модулю структур порядка малости $t^{2}$, имеет вид $\left.\| \nabla_{0}, \nabla^{\prime}\right]=0$. Таким образом, совокупность всех общих инфинитезимальных деформаций совпадает с Ker $\partial_{\nabla} \subset \mathscr{D}_{2}$.

Аналогично,совокупность всех тривиальных инфинитезимальных деформаций есть $\operatorname{Im} \partial_{\nabla} \subset \mathscr{D}_{2}$.

Следовательно, $H_{\nabla}^{2}$ можно рассматривать как фактор-пространство касательных к общим деформациям по касательным к тривиальным деформациям или, другими словами, $H_{\nabla}^{2}$ можно представлять себе как пространство модулей инфинитезимальных деформаций.

2.6. Операция внешнего умножения $\wedge: \mathscr{D}_{i} \otimes_{\mathbb{R}} \mathscr{D}_{j} \rightarrow \mathscr{D}_{i+j}$ индуцирует умножение в пуассоновых когомологиях:

$$
H_{\nabla}^{i} \otimes_{\mathbb{R}} H_{\nabla}^{j} \rightarrow H_{\nabla}^{i+j},
$$

где

$$
\bar{X} \wedge \bar{Y}=\overline{X \wedge Y}
$$


и через $\bar{X} \in H_{\nabla}^{i}$ мы обозначили класс когомологий коцикла $X \in \mathscr{D}_{i}$.

Относительно введенного умножения $H_{\nabla}^{*}=\bigoplus_{i \geqslant 0} H_{\nabla}^{i}$ является грассмановой алгеброй, т.е.

$$
\bar{X} \wedge \bar{Y}=(-1)^{i j} \bar{Y} \wedge \bar{X}
$$

для $\bar{X} \in H_{\nabla}^{i}, \bar{Y} \in H_{\nabla}^{j}$.

Аналогично, скобка Схоутена $[\cdot, \cdot]]: \mathscr{D}_{i} \otimes_{\mathbb{R}} \mathscr{D}_{j} \rightarrow \mathscr{D}_{i+j-1}$ индуцирует скобку в пуассоновых когомологиях:

$$
\| \cdot, \cdot]: H_{\nabla}^{i} \otimes_{\mathbb{R}} H_{\nabla}^{j} \rightarrow H_{\nabla}^{i+j-1},
$$

где

$$
\llbracket \bar{X}, \bar{Y} \rrbracket=\| \overline{X, Y} \rrbracket
$$

удовлетворяющую следующим свойствам:

b1) $\left.\llbracket \bar{X}, \bar{Y} \rrbracket=(-1)^{i j} \llbracket \bar{Y}, \bar{X}\right]$;

b2) $\llbracket[\bar{X}, \bar{Y} \wedge \bar{Z}]=(-1)^{k}\left\|[\bar{X}, \bar{Y}] \wedge \bar{Z}+(-1)^{i j} \bar{Y} \wedge\right\| \bar{X}, \bar{Z} \rrbracket$;

b3) $[[\bar{X}, \| \bar{Y}, \bar{Z}]]\rfloor=(-1)^{k+1}|[[\bar{X}, \bar{Y}] \mid \bar{Z}]|+(-1)^{i j+1}[\bar{Y}, \| \bar{X}, \bar{Z}] \rrbracket \mid$

для $\bar{X} \in H_{\nabla}^{i}, \bar{Y} \in H_{\nabla}^{j}, \bar{Z} \in H_{\nabla}^{k}$.

В частности, пуассоновы когомологии $H_{\nabla}^{i}, i \geqslant 0$, являются модулями над алгеброй функций Казимира $H_{\nabla}^{0}$ относительно умножения (2), а также модулями относительно скобки (3) над алгеброй Ли $H_{\nabla}^{1}$.

\section{§ 3. Формальная классификация пуассоновых структур}

3.1. Рассмотрим действие псевдогрупшы локальных диффеоморфизмов, сохраняющих фиксированную точку $m \in M$, на множестве всех пуассоновых структур.

Обозначим через $\mu \subset A$ максимальный идеал, отвечающий точке $m$ :

$$
\mu=\{f \in A \mid f(m)=0\}
$$

а через $\mu^{k}, k \geqslant 1,-$ идеал функций порядка малости $k$ в точке $m$. Соответственно через $\mu^{k} \mathscr{D}_{j}$ обозначим подмодуль $j$-векторных полей, имеющих в точке $m$ порядок малости $k$.

ОПРеДЕЛЕНИЕ 1. Скажем, что две пуассоновы структуры $\nabla$ и $\nabla_{1}$ локально әквивалентны в точке $m \in M$, если сушествует локальньй диффеоморфизм $\varphi$ некоторой окрестности точки $m$, сохраняющий эту точку и такой, что $\varphi_{*}^{(2)}(\nabla)=\nabla_{1}$.

ОПРЕДЕЛЕНИЕ 2. Будем говорить, что пуассоновы структуры $\nabla$ и $\nabla_{1}$ формально эквивалентны в точке $m$, если найдется такой локальньй диффеоморфизм $\varphi$, что $\varphi(m)=m$ и $\varphi_{*}^{(2)}(\nabla)-\nabla_{1} \in \mu^{\infty} \mathscr{D}_{2}$.

Известно, что теорема С. Ли дает локальную классификацию пуассоновых структур локально постоянного ранга. А именно, любые две такие пуассоновы структуры данного ранга $2 k$-локально эквивалентны.

Это означает, что в окрестности точки $m$ можно выбрать такие координаты $(q, p, z)$, что

$$
\nabla=\sum_{i=1}^{k} \frac{\partial}{\partial q^{i}} \wedge \frac{\partial}{\partial p^{i}} .
$$


3.2. Мы будем рассматривать формальную классификацию пуассоновых структур в том случае, когда ранг $\nabla$ не является локально постоянным. Более того, в силу “теоремы о расщеплении" А. Вейнстейна [6] мы будем предполагать, что $\nabla_{m}=0$.

Для таких структур $\theta=\nabla \bmod \mu^{2} \mathscr{D}_{2}$ определяет структуру алгебры Ли на $T_{m}^{*}$, поэтому задача локальной классификации этих структур содержит в качестве подзадачи задачу классификации всех структур алгебр Ли на $T_{m}^{*}$ относительно групшы линейных преобразований $\mathrm{GL}\left(T_{m}^{*}\right)$.

В силу этого замечания мы ограничимся классификацией пуассоновых структур по модулю классификации алгебр Ли на $T_{m}^{*}$, т.е. мы будем рассматривать классификацию пуассоновых структур относительно группы таких локальных диффеоморфизмов $\varphi: M \rightarrow M$, что $\varphi(m)=m$ и $\varphi_{*, m}=\mathrm{id}: T_{m} \rightarrow T_{m}$.

Мы применим гомотопический метод (или метод накрывающего пути) для вычисления препятствий к эквивалентности. Рассмотрим две пуассоновы структуры $\nabla$ и $\nabla_{1}$, и пусть $\nabla_{t}-$ путь в пуассоновых структурах, соединяющий $\nabla$ и $\nabla_{1} \bmod \mu^{\infty} \mathscr{D}_{2}$.

Будем искать такой путь $\varphi_{t}$ в локальных диффеоморфизмах, что

1) $\varphi_{0}=\mathrm{id}$

2) $\varphi_{t}(m)=m, 0 \leqslant t \leqslant 1$

3) $\left(\varphi_{t}\right)_{*}^{(2)}\left(\nabla_{t}\right)=\nabla \bmod \mu^{\infty} \mathscr{D}_{2}, 0 \leqslant t \leqslant 1$.

Дифференцируя соотношение 3 ) по $t$, получим эквивалентное условие:

$$
\frac{d}{d t}\left(\left(\varphi_{t}\right)_{*}^{(2)}\left(\nabla_{t}\right)\right)=0 \bmod \mu^{\infty} \mathscr{D}_{2}
$$

Затем, вычисляя эту производную, получаем следующее гомологическое уравнение на однопараметрическое семейство векторных полей $X_{t}$ :

$$
\| \nabla, X_{t} \rrbracket=\left(\dot{\sigma}_{t}-\llbracket \sigma_{t}, X_{t} \rrbracket\right) \bmod \mu^{\infty} \mathscr{D}_{2}
$$

или

$$
\partial_{\nabla}\left(X_{t}\right)=\left(\dot{\sigma}_{t}-\llbracket \sigma_{t}, X_{t} \rrbracket\right) \bmod \mu^{\infty} \mathscr{D}_{2},
$$

где $X_{t, m}=0, \sigma_{t}=\nabla_{t}-\nabla \in \mu^{p+1} \mathscr{D}_{2}, p \geqslant 1$.

Заметим, что $\partial_{\nabla}\left(\mu^{p} \mathscr{D}_{j}\right) \subset \mu^{p} \mathscr{D}_{j+1}, p \geqslant 0, j \geqslant 0$, и $\llbracket \sigma_{t}, X_{t} \rrbracket \in \mu^{p+q+1} \mathscr{D}_{2}$, если $\sigma_{t} \in \mu^{p+1} \mathscr{D}_{2}, X_{t} \in \mu^{q+1} \mathscr{D}$.

Поэтому $\llbracket \sigma_{t}, X_{t} \rrbracket$ имеет больший порядок малости, чем $\dot{\sigma}_{t}$, если $q \geqslant 1$.

Будем искать решение гомологического уравнения в классевекторньх полей $X_{t}$, имеющих в точке $m$ нуль порядка $p+1$. Тогда $\partial_{\nabla}\left(X_{t}\right)$ также имеет нуль порядка $p+1, \mathrm{a}$ гомологическое уравнение, рассматриваемое с точностью до малых $p+2$-го порядка, примет вид

$$
\partial_{\nabla}\left(X_{t}\right)=\dot{\sigma}_{t} \bmod \mu^{p+2} \mathscr{D}_{2}
$$

Обозначим через $\left[X_{t}\right]_{p+1}:=X_{t} \bmod \mu^{p+2} \mathscr{D}_{1}$ и $\left[\dot{\sigma}_{t}\right]_{p+1}:=\dot{\sigma}_{t} \bmod \mu^{p+2} \mathscr{D}_{2}$ соответствуюшие классы смежности. Тогда, используя изоморфизмы

$$
\mu^{p+1} \mathscr{D}_{j} / \mu^{p+2} \mathscr{D}_{j} \simeq S^{p+1} T_{m}^{*} \otimes_{\mathbb{R}} \Lambda^{j} T_{m}
$$

получаем следующие включения: $\left[\dot{\sigma}_{t}\right]_{p+1} \in S^{p+1} T_{m}^{*} \otimes \Lambda^{2} T_{m}$ и $\left[X_{t}\right]_{p+1} \in S^{p+1} T_{m}^{*} \otimes T_{m}$. 
3.3. Для решения уравнения (4) воспользуемся тем, что дифференциал $\partial_{\nabla}: \mathscr{D}_{j} \rightarrow$ $\mathscr{D}_{j+1}$, где $j \geqslant 0$, сохраняет порядок малости, и рассмотрим фактор-комплекс

$$
0 \rightarrow S^{p} T_{m}^{*} \stackrel{\delta}{\rightarrow} S^{p} T_{m}^{*} \otimes T_{m} \stackrel{\delta}{\rightarrow} S^{p} T_{m}^{*} \otimes \Lambda^{2} T_{m} \stackrel{\delta}{\rightarrow} \cdots
$$

где $\delta\left([X]_{p}\right):=\left[\partial_{\nabla}(X)\right]_{p}$ для всех $X \in \mu^{p} \mathscr{D}_{j}, j=0,1, \ldots$ Тогда гомологическое уравнение (4) примет вид $\delta\left(\left[X_{t}\right]_{p+1}\right)=\left[\dot{\sigma}_{t}\right]_{p+1}$. Отметим также, что оператор $\delta$ определяется классом $\theta:=[\nabla]_{1}$, поскольку для всех $\varepsilon \in \mu^{2} \mathscr{D}_{2}$

$$
\llbracket \nabla+\varepsilon, X \rrbracket=\| \nabla, X]+\llbracket \varepsilon, X]=\llbracket \nabla, X] \bmod \mu^{p+1} \mathscr{D}_{j+1}
$$

при условии, что $X \in \mu^{p+1} \mathscr{D}_{j}$.

Элемент $\theta \in T_{m}^{*} \otimes \Lambda^{2} T_{m}$ можно рассматривать как кососимметрическое отображение $\theta: \Lambda^{2} T_{m}^{*} \rightarrow T_{m}^{*}$, где $\theta(a \wedge b)=\langle\theta, a \wedge b\rangle, a, b \in T_{m}^{*}$.

Иначе говоря, если $a=d_{m} f, b=d_{m} g$, где функции $f$ и $g$ таковы, что $f(m)=g(m)=0$, то $\theta\left(d_{m} f \wedge d_{m} g\right)=d_{m}\langle\nabla, d f \wedge d g\rangle$. Обозначим через $[a, b]_{\theta}$ образ $\theta(a \wedge b) \in T_{m}^{*}$.

Теорема [6]. Пусть $\nabla \in \mathscr{D}_{2}-$ такая пуассонова структура, что $\nabla_{m}=0$. Тогда отобрахсение $\theta=[\nabla]_{1}: \Lambda^{2} T_{m}^{*} \rightarrow T_{m}^{*}$ определяет структуру алгебры Ли на кокасательном пространстве.

3.4. Обозначим через $\mathscr{G}=\left(T_{m}^{*}, \theta\right)$ алгебру Ли предыдущей теоремы.

Рассмотрим $S^{p} \mathscr{G}=S^{p} T_{m}^{*}$ как $\mathscr{G}$-модуль относительно следующего действия:

$$
b\left(a_{1} \circ \cdots \circ a_{p}\right)=\sum_{i=1}^{p} a_{1} \circ \cdots \circ a_{i-1} \circ b\left(a_{i}\right) \circ a_{i+1} \circ \cdots \circ a_{p}
$$

где $a_{i} \in T_{m}^{*}, i=1, \ldots, p, b \in T_{m}^{*}, \mathrm{a} b\left(a_{i}\right)=a d_{b}\left(a_{i}\right)=\left[a_{i}, b\right]_{\theta}$ - присоединенное действие алгебры Ли $\mathscr{G}$.

Рассматривая элементы из $S^{p} \mathscr{G} \otimes \Lambda^{j} \mathscr{G} *$ как внешние $j$-формы на алгебре Ли $\mathscr{G}$ со значениями в $\mathscr{G}$-модуле $S^{p} \mathscr{G}$, получаем следующую теорему.

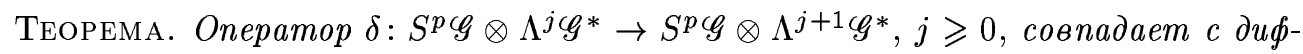
ференииалом в стандартном комплексе для когомологий алгебры Ли $\mathscr{G}$ с коэффиииентами в $\mathscr{G}$-модуле $S^{p} \mathscr{G}:$

$$
0 \rightarrow S^{p} \mathscr{G} \stackrel{\delta}{\rightarrow} S^{p} \mathscr{G} \otimes \mathscr{G}^{*} \stackrel{\delta}{\rightarrow} S^{p} \mathscr{G} \otimes \Lambda^{2} \mathscr{G}^{*} \stackrel{\delta}{\rightarrow} \cdots
$$

3.5. Воспользуемся приведенным описанием комплекса (5) для исследования гомологического уравнения.

ТЕОРема 1. Пусть бивекторное поле $\sigma \in \mu^{p+1} \mathscr{D}_{2}, p \geqslant 1$, таково, что $\nabla+\sigma$ является пуассоновой структурой. Тогда $[\sigma]_{p+1}-\delta$-коцикл.

Обратно, если $\sigma \in \mu^{p+1}, p \geqslant 1$, является $\delta$-коииклом, то

$$
\llbracket \nabla+\sigma, \nabla+\sigma]=0 \bmod \mu^{p+2} \mathscr{D}_{3}(M)
$$


ПРЕДЛОЖЕНИЕ. Класс $[\sigma]_{p+1}, \sigma \in \mu^{p+1} \mathscr{D}_{2}, p \geqslant 1$, является $\delta$-коииклом тогда $u$ только тогда, когда

$$
\llbracket \nabla+\sigma, \nabla+\sigma]=0 \bmod \mu^{p+2} \mathscr{D}_{3}(M)
$$

Таким образом, разрешимость гомологического уравнения $\delta\left(\left[X_{t}\right]_{p+1}\right)=\left[\dot{\sigma}_{t}\right]_{p+1}$ означает, что $\left[\dot{\sigma}_{t}\right]_{p+1}$ является кограницей, и препятствия к решению гомологического уравнения в $\mu^{p+1} \mathscr{D}_{1}, p \geqslant 1$, лежат в групе когомологий $H^{2}\left(\mathscr{G}, S^{p+1} \mathscr{G}\right)$.

Суммируя сказанное, получаем следуюший результат.

Теорема 2. 1) Если $H^{2}\left(\mathscr{G}, S^{p+1} \mathscr{G}\right)=0, p \geqslant 1$, то любая пуассонова структура $\nabla_{1}$ такая, что $\nabla-\nabla_{1} \in \mu^{2} \mathscr{D}_{2}$, формально әквивалентна $\nabla$.

2) Если класс $[\nabla]_{1}$ таков, что когомологии $H^{2}\left(\mathscr{G}, S^{p+1} \mathscr{G}\right)=0, p \geqslant 1$, то пуассонова структура $\nabla$ формально әквивалентна линейной пуассоновой структуре, m.e.

$$
\nabla=\sum_{i<j}\left(c_{i j}^{k} x_{k}\right) \frac{\partial}{\partial x_{i}} \wedge \frac{\partial}{\partial x_{j}}
$$

в некоторой системе локальных координат $x_{1}, \ldots, x_{n}, x_{i}(m)=0, i=1, \ldots, n$, дде $c_{i j}^{k}$ - структурные константы алгебры Ли $\mathscr{G}$.

СледСТВИЕ 1. Если $\nabla \in \mathscr{D}_{2}, \nabla_{m}=0$, - такая пуассонова структура, что $\left(T_{m}^{*}, \theta\right)$ является полупростой алгеброй Ли, то $\nabla$ формально әквивалентна линейной пуассоновой структуре.

Тем самым, мы получаем теорему А. Вейнстейна о формальной линеаризации пуассоновой структуры в случае полупростой алгебры Ли [6].

ЗАмЕчАниЕ 1. Д. Конн в статье [7] доказал, что для полупростой алгебры $\mathscr{G}$ компактного типа существует в окрестности нуля такая система локальных координат $\left(y_{1}, \ldots, y_{n}\right)$ класса $C^{\infty}$, что пуассонова структура $\nabla$ может быть записана в следующем виде:

$$
\nabla=\frac{1}{2} \sum_{1 \leqslant i, j, k \leqslant n} c_{i j}^{k} y_{k} \frac{\partial}{\partial y_{i}} \wedge \frac{\partial}{\partial y_{j}} .
$$

Аналогичньй результат получен им для аналитической пуассоновой структуры $\nabla$ с полупростой алгеброй Ли $\mathscr{G}$ и сфформулирован в работе [8].

СлЕДСТВИЕ 2. Пусть $\left(T_{m}^{*}, \theta\right)$ является редуктивной алгеброй Ли с одномерным иентром. Тогда пуассонова структура $\nabla$ формально әквивалентна линейной.

В частности, если $\left(T_{m}^{*}, \theta\right)$ - одна из следующих алгебр: $\operatorname{gl}(n), \operatorname{csp}(n), \operatorname{cso}(n), m o$ $\nabla$ формально линеаризуема.

Пусть пуассонова структура $\nabla^{\prime}$ такова, что $\nabla-\nabla^{\prime} \in \mu^{2} \mathscr{D}_{2}$.

ОПРЕДЕЛЕНИЕ. Класс когомологий $h \in H^{2}\left(\mathscr{G}, S^{p+1} \mathscr{G}\right)$ назовем допycmuмы.м omносительно $\nabla^{\prime}$, если существует бивекторное поле $\sigma \in \mu^{p+1} \mathscr{D}_{2}$ такое, что класс когомологий $[\sigma]_{p+1}$ совпадает с $h$, a $\nabla^{\prime}+\sigma$ является (формальной) пуассоновой структурой.

Определим дерево пуассоновых структур. 
Вершина - линейная пуассонова структура $\nabla^{1}=[\nabla]_{1}$.

Уровень 1 - пуассоновы структуры вида $\nabla^{1}+\sigma_{2}$, где $\sigma_{2} \in \mu^{2} \mathscr{D}_{2}$, а класс $\left[\sigma_{2}\right]_{2}$ в $H^{2}\left(\mathscr{G}, S^{2} \mathscr{G}\right)$ пробегает все $\nabla^{1}$-допустимые структуры. Для каждого $\nabla^{1}$-допустимого класса $\lambda_{1} \in H^{2}\left(\mathscr{G}, S^{2} \mathscr{G}\right)$ зафиксируем одну из таких структур и обозначим ее через $\nabla_{\lambda_{1}}^{2}$.

Уровень 2 - пуассоновы структуры вида $\nabla_{\lambda_{1}}^{2}+\sigma_{3}$, где $\sigma_{3} \in \mu^{3} \mathscr{D}_{2}$, а класс $\left[\sigma_{3}\right]_{3}$ в $H^{2}\left(\mathscr{G}, S^{3} \mathscr{G}\right)$ пробегает все $\nabla_{\lambda_{1}}^{2}$-допустимые структуры. Для каждого $\nabla_{\lambda_{1}}^{2}$-допустимого класса $\lambda_{2} \in H^{2}\left(\mathscr{G}, S^{3} \mathscr{G}\right)$ зафиксируем одну из таких структур и обозначим ее через $\nabla_{\lambda_{1}, \lambda_{2}}^{3}$.

Уровень $p$ - пуассоновы структуры вида $\nabla_{\lambda_{1}, \ldots, \lambda_{p-1}}^{p}+\sigma_{p+1}$, где $\sigma_{p+1} \in \mu^{p+1} \mathscr{D}_{2}$, а класс $\left[\sigma_{p+1}\right]_{p+1}$ в $H^{2}\left(\mathscr{G}, S^{p+1} \mathscr{G}\right)$ пробегает все $\nabla_{\lambda_{1}, \ldots, \lambda_{p-1}}^{p}$-допустимые структуры. Для каждого $\nabla_{\lambda_{1}, \ldots, \lambda_{p-1}}^{p}$-допустимого класса $\lambda_{p} \in H^{2}\left(\mathscr{G}, S^{p+1} \mathscr{G}\right)$ зафиксируем одну из таких структур и обозначим ее через $\nabla_{\lambda_{1}, \ldots, \lambda_{p}}^{p+1}$.

ПРЕДЛОЖЕНИЕ. Для любой пуассоновой структуры $\nabla^{\prime}$ такой, что $\nabla-\nabla^{\prime} \in$ $\mu^{2} \mathscr{D}_{2}$, июбого числа $p \geqslant 2$ существуют последовательность диффеоморфизмов $\varphi_{j}$, где $2 \leqslant j \leqslant p u \varphi_{j}^{*}=\mathrm{id} \bmod \mu^{j}$, и пуассонова структура $\nabla_{\lambda_{1}, \ldots, \lambda_{p-1}}^{p}$ такие, что $\varphi_{*}^{(2)}\left(\nabla^{\prime}\right)=\nabla_{\lambda_{1}, \ldots, \lambda_{p-1}}^{p} \bmod \mu^{p+1} \mathscr{D}_{2} u \varphi=\varphi_{p} \circ \cdots \circ \varphi_{2}$.

Рассмотрим два случая.

А) Дерево пуассоновых структур обрывается на некотором уровне $p_{0}$, если вьполнено $H^{2}\left(\mathscr{G}, S^{p+1} \mathscr{G}\right)=0$ при $p \geqslant p_{0} \geqslant 1$.

Теорема 3. Если $H^{2}\left(\mathscr{G}, S^{p+1} \mathscr{G}\right)=0, p \geqslant p_{0} \geqslant 1$, то $\nabla$ формально әквивалентна структуре вида

$$
\nabla=\sum_{\substack{1 \leqslant|\sigma| \leqslant p_{0} \\ i<j}} a_{i j}^{\sigma} x^{\sigma} \frac{\partial}{\partial x_{i}} \wedge \frac{\partial}{\partial x_{j}} .
$$

Здеcb

$$
\nabla^{\prime}=\sum_{\substack{|\sigma|=p+1 \\ i<j}} a_{i j}^{\sigma} x^{\sigma} \frac{\partial}{\partial x_{i}} \wedge \frac{\partial}{\partial x_{j}}, \quad p \geqslant 1,
$$

- представители допустимого относительно пуассоновой структурь

$$
\nabla^{\prime \prime}=\sum_{\substack{|\sigma| \leqslant p \\ i<j}} a_{i j}^{\sigma} x^{\sigma} \frac{\partial}{\partial x_{i}} \wedge \frac{\partial}{\partial x_{j}}
$$

класса когомологий в $H^{2}\left(\mathscr{G}, S^{p+1} \mathscr{G}\right)$.

В) В общем случае получаем следующую теорему.

Теорема 4. Пусть $\gamma_{i}^{p} \in \mu^{p+1} \mathscr{D}_{2}, i=1, \ldots, n_{p},-$ такие бивекторные поля, что образы классов $\left[\gamma_{i}^{p}\right]_{p+1}$ образуют базис в группе когомологий $H^{2}\left(\mathscr{G}, S^{p+1} \mathscr{G}\right), p \geqslant 1$.

Тогда любая пуассонова структура $\nabla$ такая, что $\nabla_{m}=0 u[\nabla]_{1}=\theta$ определяет алгебру Ли $\mathscr{G}$, формально әквивалентна следующей пуассоновой структуре:

$$
\nabla_{1}=\sum_{i<j}\left(c_{i j}^{k} x_{k}\right) \frac{\partial}{\partial x_{i}} \wedge \frac{\partial}{\partial x_{j}}+\sum_{s, p} c_{s}^{p} \gamma_{s}^{p},
$$

где $c_{i j}^{k}$ - структурные константы алгебры Ли $\mathscr{G}$, a $c_{s}^{p} \in \mathbb{R}$. 
ЗАмЕчАниЕ 2. Здесь, как и в предыдущей теореме, $\sum_{s} c_{s}^{p} \gamma_{s}^{p}$-представители допустимых относительно пуассоновых структур

$$
\sum_{i<j}\left(c_{i j}^{k} x_{k}\right) \frac{\partial}{\partial x_{i}} \wedge \frac{\partial}{\partial x_{j}}+\sum_{s, j<p} c_{s}^{j} \gamma_{s}^{j}
$$

классов когомологий в $H^{2}\left(\mathscr{G}, S^{p+1} \mathscr{G}\right)$.

\section{§4. Спектральная последовательность}

В этом параграфе мы построим спектральную последовательность, которая дает более подробное описание нормальных форм пуассоновых структур.

4.1. ОПРЕДЕЛЕНИЕ 1 . Две пуассоновы структуры $\nabla$ и $\nabla_{1}$ называются $k$-әквивалентными в точке $m, k=0,1,2, \ldots$, если существует диффеоморфизм $\varphi$ некоторой окрестности этой точки, сохраняющий $m$ и такой, что $\varphi(m)=m$ и $\varphi_{*}^{(2)}(\nabla)-\nabla_{1} \in \mu^{k+1} \mathscr{D}_{2}$.

ОПРЕДЕЛЕниЕ 2. Пуассонова структура $\nabla$ назьвается $k$-достаточной, $k=0,1$, $2, \ldots$, если любая $k$-эквивалентная ей пуассонова структура $\nabla_{1} \in \mathscr{D}_{2}$ является (формально) эквивалентной $\nabla$.

Введем фильтрацию в комплекс (1), положив

$$
F_{p, q}=\mu^{p} \mathscr{D}_{p+q}
$$

Из условия $\nabla \in \mu \mathscr{D}_{2}$ следует, что $\partial_{\nabla}\left(\mu^{p} \mathscr{D}_{j}\right) \subset \mu^{p} \mathscr{D}_{j+1}$. Тем самым, дифференциал $\partial_{\nabla}$ сохраняет фильтрацию: $\partial_{\nabla}\left(F_{p, q}\right) \subset F_{p, q+1}$. Эта фильтрация также согласована со скобкой Схоутена в следуюшем смысле: $\| F_{a, b}, F_{c, d} \rrbracket \subset F_{a+c-1, b+d}$. Пусть

$$
Z_{r}^{p, q}=\left\{\omega \in F_{p, q} \mid \partial_{\nabla}(\omega) \in F_{p+r, q-r+1}\right\}
$$

- $(p, q)$-коциклы порядка $r$ и

$$
B_{r}^{p, q}=\left\{\omega \in F_{p, q} \mid \exists \theta \in F_{p-r, q+r-1}: \partial_{\nabla}(\theta)=\omega\right\}
$$

- $(p, q)$-кограницы порядка $r, B_{r}^{p, q}=\partial_{\nabla}\left(Z_{r}^{p-r, q+r-1}\right)$. Напомним, что спектральная последовательность $\left(E_{r}^{p, q}, d_{r}^{p, q}\right)$ когомологий комплекса $(1)$, построенная по фильтрации (6), имеет вид

$$
E_{r}^{p, q}=Z_{r}^{p, q} /\left(Z_{r-1}^{p+1, q-1}+B_{r-1}^{p, q}\right),
$$

а дифференциалы $d_{r}=\left(\partial_{\nabla}\right)_{r}^{p, q}: E_{r}^{p, q} \rightarrow E_{r}^{p+r, q-r+1}$ порождены $\partial_{\nabla}$.

ПРЕДЛОЖЕНИЕ. Справедливы следующие включения:

a) $\left.\llbracket Z_{r}^{p, q}, Z_{r}^{p^{\prime}}, q^{\prime}\right] \subset Z_{r}^{p+p^{\prime}-1, q+q^{\prime}}$;

b) $\left[B_{r-1}^{p, q}, Z_{r}^{p^{\prime}}, q^{\prime}\right] \subset B_{r-1}^{p+p^{\prime}-1, q+q^{\prime}}+Z_{r-1}^{p+p^{\prime}, q+q^{\prime}-1}$

c) $\|\left[Z_{r-1}^{p+1, q-1}, Z_{r}^{p^{\prime}}, q^{\prime}\right] \subset Z_{r-1}^{p+p^{\prime}, q+q^{\prime}-1}$. 
Это предложение показывает, что скобка Схоутена может быть перенесена на спектральную последовательность.

Действительно, определим спаривание

$$
E_{r}^{p, q} \times E_{r}^{p^{\prime}, q^{\prime}} \rightarrow E_{r}^{p+p^{\prime}-1, q+q^{\prime}},
$$

положив $\left.\left.\llbracket x_{r}^{p, q}, y_{r}^{p^{\prime}}, q^{\prime}\right]=\llbracket x, y\right] \bmod \left(Z_{r-1}^{p+p^{\prime}}, q+q^{\prime}-1+B_{r-1}^{p+p^{\prime}-1, q+q^{\prime}}\right)$ для классов смежности

$$
x_{r}^{p, q}=x \bmod \left(Z_{r-1}^{p+1, q-1}+B_{r-1}^{p, q}\right), \quad y_{r}^{p^{\prime}, q^{\prime}}=y \bmod \left(Z_{r-1}^{p^{\prime}+1, q^{\prime}-1}+B_{r-1}^{p^{\prime}, q^{\prime}}\right),
$$

где $x \in Z_{r}^{p, q}, y \in Z_{r}^{p^{\prime}}, q^{\prime}$.

Свойства скобки Схоутена на алгебре поливекторньх полей индуцируют соответствующие свойства скобки в спектральной последовательности:

$$
\begin{gathered}
\left.\left.\llbracket x_{r}^{p, q}, y_{r}^{p^{\prime}}, q^{\prime}\right]=(-1)^{s t} \llbracket y_{r}^{p^{\prime}, q^{\prime}}, x_{r}^{p, q}\right], \\
\left.\| x_{r}^{p, q}, \llbracket y_{r}^{p^{\prime}, q^{\prime}}, z_{r}^{p^{\prime \prime}, q^{\prime \prime}} \rrbracket \rrbracket=(-1)^{k+1} \llbracket \llbracket\left[x_{r}^{p, q}, y_{r}^{p^{\prime}}, q^{\prime}\right], z_{r}^{p^{\prime \prime}, q^{\prime \prime}} \rrbracket+(-1)^{s t+1} \llbracket y_{r}^{p^{\prime}, q^{\prime}}, \llbracket x_{r}^{p, q}, z_{r}^{p^{\prime \prime}, q^{\prime \prime}}\right] \rrbracket,
\end{gathered}
$$

где $s=p+q, t=p^{\prime}+q^{\prime}, k=p^{\prime \prime}+q^{\prime \prime}$.

Кроме того,

$$
\left.\left.\left.d_{r}\left(\llbracket x_{r}^{p, q}, y_{r}^{p^{\prime}, q^{\prime}}\right]\right)=\llbracket d_{r}\left(x_{r}^{p, q}\right), y_{r}^{p^{\prime}}, q^{\prime}\right]+(-1)^{p+q} \llbracket x_{r}^{p, q}, d_{r}\left(y_{r}^{p^{\prime}}, q^{\prime}\right)\right] .
$$

4.2. Опишем начальные члены полученной спектральной последовательности.

В случае $r=0$ имеем

$$
E_{0}^{p, q}=F_{p, q} / F_{p+1, q-1}=\mu^{p} \mathscr{D}_{p+q} / \mu^{p+1} \mathscr{D}_{p+q}=S^{p} T_{m}^{*} \otimes_{\mathbb{R}} \Lambda^{p+q} T_{m} .
$$

Из теоремы п. 3.3 следует, что дифференциал $d_{0}: E_{0}^{p, q} \rightarrow E_{0}^{p, q+1}$ совпадает с дифференциалом $\delta$ в стандартном комплексе для когомологий алгебры Ли $\mathscr{G}=\left(T_{m}^{*}, \theta\right)$ с коэффициентами в $\mathscr{G}$-модуле $S^{p} \mathscr{G}$.

Поэтому $E_{1}^{p, q}=H^{p+q}\left(\mathscr{G}, S^{p} \mathscr{G}\right)$. В частности, при $p+q=0$ имеем $E_{1}^{p,-p}=\left(S^{p} \mathscr{G}\right)^{\mathscr{G}}$, где через $L^{\mathscr{G}}$ для $\mathscr{G}$-модуля $L$ обозначено подпространство $\mathscr{G}$-инвариантных элементов $L$ :

$$
L^{\mathscr{G}}=\{x \in L \mid g(x)=0 \forall g \in \mathscr{G}\} .
$$

ТЕОРемА. Фильтрация $F_{p, q}$ определяет спектральную последовательность $\left(E_{r}^{p, q}, d_{r}\right)$, в которой

a) $E_{0}^{p, q}=S^{p} T_{m}^{*} \otimes_{\mathbb{R}} \Lambda^{p+q} T_{m}$

b) $E_{1}^{p, q}=H^{p+q}\left(\mathscr{G}, S^{p} \mathscr{G}\right)$;

c) члены $E_{r}^{p, q}$ несут структуру биградуированной алгебры Ли относительно скобки Схоутена, т.е. на $\bigoplus_{p, q} E_{r}^{p, q}$ определено билинейное спаривание

$$
E_{r}^{p, q} \times E_{r}^{p^{\prime}, q^{\prime}} \rightarrow E_{r}^{p+p^{\prime}-1, q+q^{\prime}},
$$

получаемое редукиией скобки Схоутена, которое удовлетворяет соотношениям (8), (9), а дифференциал $d_{r}$ является дифферениированием этой алгебры (10);

d) спектральная последовательность стабилизируется в следующем смысле: для каждой пары чисел $(p, q)$ найдется такое число $r_{0}=r_{0}(p, q)$, что

$$
E_{r_{0}}^{p, q}=E_{r_{0}+1}^{p, q}=\cdots=E_{\infty}^{p, q}
$$

əде $d_{r}=0, r \geqslant r_{0}$. 
4.3. Рассмотрим такие пуассоновы структуры $\nabla \in \mu \mathscr{D}_{2}$, для которых

$$
\mathscr{G}=\left(T_{m}^{*}, \theta=\nabla \bmod \mu^{2} \mathscr{D}_{2}\right)
$$

является редуктивной алгеброй Ли.

Обозначим через $\mathscr{G}_{0}=[\mathscr{G}, \mathscr{G}]$ полупростую часть и через $C$-центр алгебры $\mathscr{G}$. Тогда из теоремы п. 4.2 получаем следующее утверждение.

ТЕоремА. Для указанной выше пуассоновой структуры $\nabla$ член $E_{1}^{p, q}$ в спектральной последовательности (7) имеет вид

$$
E_{1}^{p, q}=\sum_{i+j=p+q} H^{i}\left(\mathscr{G}_{0}, \mathbb{R}\right) \otimes_{\mathbb{R}} \Lambda^{j}\left(C^{*}\right) \otimes_{\mathbb{R}}\left(S^{p} \mathscr{G}\right)^{\mathscr{G}}
$$

\section{§ 5. Нормальные формы пуассоновых структур}

5.1. Использование спектральной последовательности для получения нормальных форм пуассоновых структур основано на следующих замечаниях.

Во-первых, как и вьше, мы будем рассматривать классификацию пуассоновых структур относительно группы таких локальных диффеоморфизмов $\varphi: M \rightarrow M$, что $\varphi(m)=m$ и $\varphi_{*, m}=\mathrm{id}: T_{m} \rightarrow T_{m}$.

Во-вторьх, если $\nabla \in \mathscr{D}_{2}$ и $\nabla+\sigma \in \mathscr{D}_{2}$ являются пуассоновыми структурами, то $\sigma$ удовлетворяет следуюшему уравнению:

$$
\partial_{\nabla}(\sigma)+\frac{1}{2} \llbracket[\sigma, \sigma]=0 .
$$

Поэтому $\partial_{\nabla}(\sigma)=-\frac{1}{2} \mid[\sigma, \sigma] \in F_{2 p-1,4-2 p}$, если $\sigma \in F_{p, 2-p}$ и, следовательно, $\sigma \in Z_{r}^{p, 2-p}$ для всех $r \leqslant p-1$.

В-третьих, обозначив через $\varphi_{t}$ однопараметрическую группу локальных диффеоморфизмов, отвечающую векторному полю $X \in \mu \mathscr{D}$, мы будем использовать следующую формулу:

$$
\left(\varphi_{t}\right)_{*}^{(2)}(\nabla)=\nabla+t \|[\nabla, X]+\frac{1}{2} t^{2}|[[\nabla, X], X]|+\cdots
$$

откуда вытекает следующее

ПРЕДЛОЖЕНИЕ. Пусть $X \in Z_{r}^{p, 1-p}$. Тогда для локального диффеоморфизма $\varphi=\varphi_{1}$ имеем

$$
\varphi_{*}^{(2)}(\nabla)-\nabla-\partial_{\nabla}(X) \in F_{2 p+r-1,3-2 p-r} .
$$

5.2. Учитьвая эти замечания, получаем следующую теорему, описывающую нормальную форму $p$-го приближения.

ТЕОрема 1. Пусть бивекторные поля $v_{1}, \ldots, v_{r}, v_{i} \in \mu^{p} \mathscr{D}_{2}, p \geqslant 2$, таковы, что uх образы порождают $E_{p-s+1}^{p, 2-p}$, где $2 \leqslant s \leqslant p+1$.

Тогда для каждой пуассоновой структуры вида $\nabla+\varepsilon$, где $\varepsilon \in \mu^{p} \mathscr{D}_{2}, p \geqslant 2$, существует такой локальный диффеоморфизм $\varphi, ч т о ~ \varphi^{*}=\operatorname{id} \bmod \mu^{s} u$

$$
\varphi_{*}^{(2)}(\nabla)-\nabla-\varepsilon \equiv \sum_{i=1}^{r} c_{i} v_{i} \bmod F_{p+1,1-p},
$$

әде $c_{i} \in \mathbb{R}, 1 \leqslant i \leqslant r$. 
ТЕорема 2. В условиях теоремы 1 существует такой диффеоморфизм $\varphi$, что $\varphi^{*}=\mathrm{id} \bmod \mu^{s} u$

$$
\varphi_{*}^{(2)}\left(\nabla-\sum_{i=1}^{r} c_{i} v_{i}\right)=(\nabla+\varepsilon) \bmod F_{p+1,1-p}
$$

5.3. Последовательное применение теоремы 1 п. 5.2 приводит к следующему результату.

ТЕОРЕма 1. Зафиксируем последовательность чисел

$$
2 \leqslant s(2) \leqslant s(3) \leqslant \cdots \leqslant s(p) \leqslant \cdots,
$$

в которой имеется конечное число равенств $и$ для которой $s(p) \leqslant p+1$. Пусть әлементы $v_{1}, \ldots, v_{r_{p}} \in \mu^{p} \mathscr{D}_{2}, 1 \leqslant r_{p} \leqslant \infty$, таковы, что их образы порождают все $E_{p-s(p)+1}^{p, 2-p}, p \geqslant 2$.

Тогда для кажсдой пуассоновой структуры вида $\nabla+\varepsilon$, где $\varepsilon \in \mu^{q} \mathscr{D}_{2}, q \geqslant 2$, найдется такой локальный диффеоморфизм $\varphi$, что

$$
\varphi_{*}^{(2)}(\nabla)-\nabla-\varepsilon \equiv \sum_{i} c_{i} v_{i} \bmod \mu^{\infty} \mathscr{D}_{2}
$$

ЗАмЕчАниЕ. Выбор представителей $v_{i}$ и, что более важно, представителей $\sum_{i} c_{i} v_{i}$ для классов из $E_{p-s(p)+1}^{p, 2-p}$ не произволен, а специален: классы должны быть допустимыми относительно соответствующих пуассоновых структур.

По аналогии с рассуждениями, приведенньми в п. 3.5, построим редуцированное дерево пуассоновых структур.

Для этого фиксируем последовательность $s(p)$ и рассмотрим следующую конструкцию.

Вершина - линейная пуассонова структура $\nabla^{1}=[\nabla]_{1}$.

Уров ень 1 совпадает с первым уровнем дерева пуассоновых структур (п. 3.5), т.е. это пуассоновы структуры $\nabla_{\lambda_{1}}^{2}$, где $\lambda_{1}$ - допустимый относительно $\nabla^{1}$ класс когомологий в $H^{2}\left(\mathscr{G}, S^{2} \mathscr{G}\right)$.

Уровень 2 - пуассоновы структуры вида $\nabla_{\lambda_{1}}^{2}+\sigma_{3}$, где $\sigma_{3} \in \mu^{3} \mathscr{D}_{2}-$ представитель $\nabla_{\lambda_{1}}^{2}$-допустимых классов когомологий $\lambda_{2}$ в $E_{4-s(3)}^{3,-1}$. Здесь $E_{4-s(3)}^{3,-1}$ - член спектральной последовательности, построенной по пуассоновой структуре $\nabla_{\lambda_{1}}^{2}$. Обозначим эти структуры $\nabla_{\lambda_{1}, \lambda_{2}}^{3}$.

Уровень $p-$ пуассоновы структуры вида $\nabla_{\lambda_{1}, \ldots, \lambda_{p-1}}^{p}+\sigma_{p+1}$, где $\sigma_{p+1} \in \mu^{p+1} \mathscr{D}_{2}-$ представитель $\nabla_{\lambda_{1}, \ldots, \lambda_{p-1}}^{p}$-допустимых классов когомологий $\lambda_{p}$ в $E_{p-s(p+1)+2}^{p+1,1-p}$. Здесь $E_{p-s(p+1)+2}^{p+1,1-p}-$ член спектральной последовательности, построенной по пуассоновой структуре $\nabla_{\lambda_{1}, \ldots, \lambda_{p-1}}^{p}$. Обозначим эти структуры $\nabla_{\lambda_{1}, \ldots, \lambda_{p}}^{p+1}$.

Введем также понятие ветви дерева (формальных) пуассоновых структур. Рассмотрим возникающие в дереве последовательности пуассоновых структур вида

$$
\nabla^{1}, \nabla_{\lambda_{1}}^{2}, \nabla_{\lambda_{1}, \lambda_{2}}^{3}, \ldots, \nabla_{\lambda_{1}, \ldots, \lambda_{p-1}}^{p}, \ldots
$$


Каждую такую последовательность назовем ветвью дерева. Для каждой ветви сушествует (формальная) пуассонова структура $\widetilde{\nabla}, p$-эквивалентная $p$-й точкеветвления ветви $\left((p-1)\right.$-му уровню ветви) - пуассоновой структуре $\nabla_{\lambda_{1}, \ldots, \lambda_{p-1}}^{p}$.

$\Phi$ иксируем структуру такого вида и обозначим ее через $\nabla_{\left\{\lambda_{i}\right\}_{1}^{\infty}}$. Будем отождествлять ветви и задающие их пуассоновы структуры.

Теорема 2. 1) Любая пуассонова структура вида $\nabla+\varepsilon$, әде $\varepsilon \in \mu^{2} \mathscr{D}_{2}$, формально эквивалентна одной из ветвей приведенного дерева, т.е. существует диффеоморфизм ч такой, что

$$
\varphi_{*}^{(2)}(\nabla+\varepsilon)=\nabla_{\left\{\lambda_{i}\right\}_{1}^{\infty} \bmod \mu^{\infty} \mathscr{D}_{2}}
$$

для некоторой последовательности $\left\{\lambda_{i}\right\}_{1}^{\infty}$.

В частности, $\nabla+\varepsilon$ р-эквивалентна пуассоновой структуре $\nabla_{\lambda_{1}, \ldots, \lambda_{p-1}}^{p}$ из $(p-1)$-го уровня дерева.

2) Две пуассоновы структуры, формально әквивалентные одной ветви дерева, формально әквивалентны.

\section{§6. Алгебры Ли-Склянина}

6.1. В этом параграфе мы рассмотрим случай, когда алгебра Ли $\mathscr{G}=\left(T_{m}^{*}, \theta\right)$ коммутативна. Тогда $\nabla \in \mu^{2} \mathscr{D}_{2}$.

Имеем $E_{0}^{p, q}=S^{p} \mathscr{G} \otimes_{\mathbb{R}} \Lambda^{p+q} \mathscr{G}^{*}, d_{0}^{p, q}=0$. Поэтому $E_{1}^{p, q}=E_{0}^{p, q}=S^{p} \mathscr{G}_{\mathbb{R}} \Lambda^{p+q} \mathscr{G}^{*}$. Рассмотрим дифференциал

$$
d_{a, b}^{1}=d_{a, b}: S^{a} \mathscr{G} \otimes \Lambda^{b} \mathscr{G}^{*} \rightarrow S^{a+1} \mathscr{G} \otimes \Lambda^{b+1} \mathscr{G}^{*} .
$$

Заметим, что $d_{a, b}^{1}$ определяется классом $\nabla \bmod \mu^{3} \mathscr{D}_{2}$. Действительно, пусть

$$
[X]_{a}=X \bmod \mu^{a+1} \mathscr{D}_{b} \in S^{a} \mathscr{G} \otimes \Lambda^{b} \mathscr{G}^{*}
$$

для некоторого $X \in \mu^{a} \mathscr{D}_{b}$.

Тогда $\left.d_{a, b}^{1}\left([X]_{a}\right)=\llbracket X, \nabla\right] \bmod \mu^{a+2} \mathscr{D}_{b+1}$, так как $\left.\| X, \varepsilon\right] \in \mu^{a+2} \mathscr{D}_{b+1}$, если $\varepsilon \in \mu^{3} \mathscr{D}_{2}$. Обозначим $\theta=[\nabla]_{2} \in S^{2} T_{m}^{*} \otimes \Lambda^{2} T_{m}$.

Этот элемент можно рассматривать как оператор $\theta: T_{m}^{*} \wedge T_{m}^{*} \rightarrow S^{2} T_{m}^{*}$, где

$$
p \wedge q \mapsto[p, q]_{\theta}:=\langle\theta, p \wedge q\rangle=\langle\nabla, d f \wedge d g\rangle \bmod \mu^{3}
$$

для $p=d_{m} f, q=d_{m} g$ и $f(m)=g(m)=0$.

6.2. Как и в случае пары $\left(T_{m}^{*},[\nabla]_{1}\right)$, определяющей алгебру Ли на $T_{M}^{*}$, рассмотренная вьше пара $\left(T_{m}^{*},[\nabla]_{2}\right)$ не произвольна, а определяет некоторую структуру на $T_{m}^{*}$, которую мы называем алгеброй Ли-Склянина.

Для того чтобы сформулировать условия для $\theta=[\nabla]_{2}$, заметим, что скобка Схоутена $\left[\mathscr{D}_{i}, \mathscr{D}_{j}\right] \subset \mathscr{D}_{i+j-1}$ обладает следующим свойством:

$$
\left\|\mu^{p} \mathscr{D}_{i}, \mu^{q} \mathscr{D}_{j}\right\| \in \mu^{p+q-1} \mathscr{D}_{i+j-1}
$$

Поэтому мы можем определить новую скобку

$$
\llbracket S^{p} T_{m}^{*} \otimes \Lambda^{i} T_{m}, S^{q} T_{m}^{*} \otimes \Lambda^{j} T_{m} \rrbracket^{\prime} \subset S^{p+q-1} T_{m}^{*} \otimes \Lambda^{i+j-1} T_{m}
$$


Заметим, что в действительности скобку $[\cdot \cdot, \cdot]]^{\prime}$ :

$$
\left[\left[S^{p} E^{*} \otimes \Lambda^{i} E, S^{q} E^{*} \otimes \Lambda^{j} E\right]^{\prime} \subset S^{p+q-1} E^{*} \otimes \Lambda^{i+j-1} E\right.
$$

можно определить для любого векторного пространства $E$.

Для этого воспользуемся индукцией по $i+j$.

1) Для $i=0$ положим

$$
\begin{aligned}
\| \theta, \Phi \otimes X_{1} \wedge \cdots \wedge X_{j} \rrbracket^{\prime} & \left.=\llbracket \Phi \otimes X_{1} \wedge \cdots \wedge X_{j}, \theta\right]^{\prime} \\
& =\Phi \circ \sum_{i}(-1)^{i} X_{i}(\theta) \otimes X_{1} \wedge \cdots \wedge \widehat{X}_{i} \wedge \cdots \wedge X_{j} \\
& :=i_{\theta}\left(\Phi \otimes X_{1} \wedge \cdots \wedge X_{j}\right),
\end{aligned}
$$

где $\theta \in S^{p} E^{*}, \Phi \in S^{q} E^{*}, X_{i} \in E, 1 \leqslant i \leqslant j$.

Определим действие $E$ на пространстве $S^{p} E^{*}: E \otimes S^{p} E^{*} \rightarrow S^{p-1} E^{*}$ следующим образом:

$$
X \otimes \theta_{1} \circ \cdots \circ \theta_{p} \mapsto\left(X, \theta_{1}\right) \theta_{2} \circ \cdots \circ \theta_{p}+\cdots+\theta_{1} \circ \cdots \circ \theta_{p-1}\left(X, \theta_{p}\right) .
$$

Продолжим определение скобки $[[\cdot, \cdot]]^{\prime}$.

2) Если $i=1, j=1$, то положим

$$
\| \theta \otimes X, \Phi \otimes Y]^{\prime}=\theta \circ X(\Phi) \otimes Y-\Phi \circ Y(\theta) \otimes X,
$$

где $\theta \in S^{p} E^{*}, X, Y \in E, \Phi \in S^{q} E^{*}$.

3) Если $X \in S^{q} E^{*} \otimes \Lambda^{i} E, Y \in S^{q^{\prime}} E^{*} \otimes \Lambda^{j} E$, то элемент

$$
\| X, Y]^{\prime} \in S^{q+q^{\prime}-1} E^{*} \otimes \Lambda^{i+j-1} E
$$

таков, что $i_{\theta}\left([X, Y]^{\prime}\right)=\llbracket\left[i_{\theta}(X), Y\right]^{\prime}+(-1)^{i}\left[X, i_{\theta}(Y)\right]^{\prime}$ для всех $\theta \in S^{p} E^{*}$.

ПРЕДЛОЖенИЕ. СКобка $\llbracket \cdot, \cdot \cdot]^{\prime}$ удовлетворяет следующим свойствам:

a) $\| X, Y]^{\prime}=(-1)^{i j}[Y, X]^{\prime}$

b) $\left[[X, Y \wedge Z]^{\prime}=(-1)^{k}\left[[X, Y]^{\prime} \wedge Z+(-1)^{i j} Y \wedge \|[X, Z]^{\prime}\right.\right.$;

c) $\left[\left[X,[Y, Z]^{\prime}\right]^{\prime}=(-1)^{k+1}\left[\left[[X, Y]^{\prime}, Z\right]^{\prime}+(-1)^{i j+1}\left[Y,\left[[X, Z]^{\prime}\right]^{\prime}\right.\right.\right.$,

где $X \in S^{p} E^{*} \otimes \Lambda^{i} E, Y \in S^{q} E^{*} \otimes \Lambda^{j} E, Z \in S^{r} E^{*} \otimes \Lambda^{k} E$.

6.3. ОпредЕлЕниЕ. Скажем, что элемент $\theta \in S^{2} E^{*} \otimes \Lambda^{2} E$ определяет структуру алгебры Ли-Склянина на пространстве $E$, если $\| \theta, \theta]^{\prime}=0$.

ПримеР. Пусть $E=\mathbb{R}^{4}$, а $x_{1}, x_{2}, x_{3}, x_{4}-$ базис в $E^{*}$.

Тогда элемент $\theta \in S^{2} E^{*} \otimes \Lambda^{2} E$ :

$$
\begin{aligned}
\theta= & -x_{1} x_{2} \frac{\partial}{\partial x_{1}} \wedge \frac{\partial}{\partial x_{2}}-x_{1} x_{3} \frac{\partial}{\partial x_{1}} \wedge \frac{\partial}{\partial x_{3}}-2 x_{2} x_{3} \frac{\partial}{\partial x_{1}} \wedge \frac{\partial}{\partial x_{4}} \\
& -x_{2} x_{4} \frac{\partial}{\partial x_{2}} \wedge \frac{\partial}{\partial x_{4}}-x_{3} x_{4} \frac{\partial}{\partial x_{3}} \wedge \frac{\partial}{\partial x_{4}}
\end{aligned}
$$

определяет структуру алгебры Ли-Склянина на $\mathbb{R}^{4}[4]$. 
Используем построенную вьше спектральную последовательность для определения когомологий Ли-Склянина.

Для этого рассмотрим последовательность

$$
\begin{aligned}
0 \longrightarrow S^{k} E^{*} & \stackrel{\partial_{\theta}}{\longrightarrow} S^{k+1} E^{*} \otimes E \stackrel{\partial_{\theta}}{\longrightarrow} \cdots \stackrel{\partial_{\theta}}{\longrightarrow} S^{k+i} E^{*} \otimes \Lambda^{i} E \\
& \stackrel{\partial_{\theta}}{\longrightarrow} S^{k+i+1} E^{*} \otimes \Lambda^{i+1} E \stackrel{\partial_{\theta}}{\longrightarrow} \cdots
\end{aligned}
$$

с дифференциалом $\partial_{\theta}: S^{k+i} E^{*} \otimes \Lambda^{i} E \rightarrow S^{k+i+1} E^{*} \otimes \Lambda^{i+1} E$, действующим следуюшим образом: $\partial_{\theta}(X)=(-1)^{i}[X, \theta]^{\prime}$, где $X \in S^{k+i} E^{*} \otimes \Lambda^{i} E$.

ПРЕДЛОЖЕНИЕ. Пара $\left(T_{m}^{*}, \theta\right)$ определяет структуру алгебры Ли-Склянина тогда и только тогда, когда $\partial_{\theta}^{2}=0$.

ОПРЕДЕЛЕНИЕ. Когомологии комплекса (11) в члене $S^{k+i} E^{*} \otimes \Lambda^{i} E$ обозначим через $H^{k+i, i}(E, \theta)$ и назовем $(k+i, i)$-когомологиями алгебры Ли-Склянина.

Суммируя сказанное вьше, получаем следующий результат.

Теорема. Пусть $\nabla \in \mu^{2} \mathscr{D}_{2}$ - пуассонова структура. Тогда пара $\left(T_{m}^{*}, \theta\right)$, әде $\theta=\nabla \bmod \mu^{3} \mathscr{D}_{2}$, определяет структуру алгебры Ли-Склянина на $T_{m}^{*}, a$ второй член спектральной последовательности $\left(E_{2}^{p, q}, d_{2}^{p, q}\right)$ совпадает $c$ әруппой $(p+q, p)$-когомологий алгебры Ли-Склянина $E_{2}^{p, q}=H^{p+q, p}\left(T_{m}^{*}, \theta\right)$.

Московский институт радиоэлектроники и автоматики

Поступило

E-mail: lychagin@glas.apc.org

22.01 .96

\section{СПИСОК ЦИТИРОВАННОЙ ЛИТЕРАТУРЫ}

[1] Арнольд В.И. Спектральные последовательности для приведения функций к нормальным формам // Задачи механики и матем. физики. М.: Наука, 1976. С. 7-20.

[2] Лычагин В.В. Особенности решений, спектральные последовательности и нормальные формы алгебр Ли векторных полей // Изв. АН СССР. Сер. матем. 1987. Т. 51. № 3. С. 584-612.

[3] Lichnerowicz A. Les varietes de Poisson et leurs algebres de Lie associees // J. Diff. Geom. 1977. V. 12. P. 253-300.

[4] Карасев М. В., Маслов В. П. Нелинейные скобки Пуассона. Геометрия и квантование. M.: Наука, 1991.

[5] Brylinski J. L. A differential complex for Poisson manifolds // J. Diff. Geom. 1988. V. 28. P. 93-114.

[6] Weinstein A. The local structures of Poisson manifolds // J. Diff. Geom. 1983. V. 18. P. 523-557.

[7] Conn J. F. Normal forms for smooth Poisson structures // Ann. of Math. 1985. V. 121. P. 565-593.

[8] Conn J. F. Normal forms for analytic Poisson structures // Ann. of Math. 1984. V. 119. P. 577-601. 